\section{OPEN ACCESS}

Edited by:

Martin M. Edreira,

Universidad de Buenos Aires, Argentina

Reviewed by:

Manuel Elkin Patarroyo, Colombian Institute of Immunology

Foundation, Colombia Gavin Wright, Wellcome Sanger Institute (WT),

United Kingdom

*Correspondence:

Jean-François Franetich jean-francois.franetich@upmc.fr

${ }^{\dagger}$ Deceased

Specialty section: This article was submitted to Parasite and Host, a section of the journal

Frontiers in Cellular and Infection Microbiology

Received: 03 May 2021 Accepted: 02 June 2021 Published: 29 June 2021

Citation:

Amanzougaghene $N$, Tajeri S, Yalaoui S, Lorthiois A, Soulard V, Gego A, Rametti A, Risco-Castillo V, Moreno A, Tefit M, van Gemert G-J,

Sauerwein RW, vaillant $J-C$,

Ravassard P, Pérignon J-L,

Froissard $P$, Mazier $D$ and

Franetich J-F (2021) The Host

Protein Aquaporin-9 Is Required

for Efficient Plasmodium

falciparum Sporozoite Entry

Into Human Hepatocytes.

Front. Cell. Infect. Microbiol. 11:704662.

doi: $10.3389 /$ fcimb.2021.704662

\title{
The Host Protein Aquaporin-9 is Required for Efficient Plasmodium falciparum Sporozoite Entry into Human Hepatocytes
}

\begin{abstract}
Nadia Amanzougaghene ${ }^{1}$, Shahin Tajeri ${ }^{1}$, Samir Yalaoui ${ }^{2,3}$, Audrey Lorthiois ${ }^{2,3}$, Valérie Soulard ', Audrey Gego ${ }^{2,3}$, Armelle Rametti $^{2,3}$, Véronica Risco-Castillo ${ }^{4}$, Alicia Moreno ${ }^{1}$, Maurel Tefit ${ }^{1}$, Geert-Jan van Gemert ${ }^{5}$, Robert W. Sauerwein ${ }^{5}$, Jean-Christophe Vaillant ${ }^{6}$, Philippe Ravassard ${ }^{7}$, Jean-Louis Pérignon ${ }^{2,3}$, Patrick Froissard ${ }^{2,3 \dagger}$, Dominique Mazier ${ }^{1}$ and Jean-François Franetich ${ }^{1 *}$
\end{abstract}

\begin{abstract}
1 Sorbonne Université, INSERM, CNRS, Centre d'Immunologie et des Maladies Infectieuses, CIMI-Paris, Paris, France, 2 Université Pierre et Marie Curie-Paris 6, UMR S945, Paris, France, ${ }^{3}$ INSERM, U945, Paris, France, ${ }^{4}$ EnvA, UPEC, Anses, Dynamyc research group EA 7380, Maisons-Alfort, France, ${ }^{5}$ Department of Medical Microbiology, Radboud University Nijmegen Medical Centre, MMB-NCMLS, Nijmegen, Netherlands, ${ }^{6}$ AP-HP, Service de Chirurgie Digestive, Hépato-BilioPancréatique et Transplantation Hépatique, Centre Hospitalo-Universitaire Pitié-Salpêtrière, Paris, France, ${ }^{7}$ CR-ICM - LGN CNRS UMR-7991, IFR des Neurosciences, Groupe Hospitalier Pitié-Salpêtrière, Paris, France
\end{abstract}

Hepatocyte invasion by Plasmodium sporozoites represents a promising target for innovative antimalarial therapy, but the molecular events mediating this process are still largely uncharacterized. We previously showed that Plasmodium falciparum sporozoite entry into hepatocytes strictly requires CD81. However, CD81-overexpressing human hepatoma cells remain refractory to $P$. falciparum infection, suggesting the existence of additional host factors necessary for sporozoite entry. Here, through differential transcriptomic analysis of human hepatocytes and hepatoma HepG2-CD81 cells, the transmembrane protein Aquaporin-9 (AQP9) was found to be among the most downregulated genes in hepatoma cells. RNA silencing showed that sporozoite invasion of hepatocytes requires AQP9 expression. AQP9 overexpression in hepatocytes increased their permissiveness to $P$. falciparum. Moreover, chemical disruption with the AQP9 inhibitor phloretin markedly inhibited hepatocyte infection. Our findings identify AQP9 as a novel host factor required for $P$. falciparum sporozoite hepatocyte-entry and indicate that AQP9 could be a potential therapeutic target.

Keywords: Plasmodium falciparum, sporozoites, liver stage, hepatocytes, Aquaporin-9, CD81

\section{INTRODUCTION}

Of the protozoan parasites of humans, Plasmodium falciparum is the deadliest, accounting for an estimated 228 million cases of malaria and causing 405000 deaths in 2018 (WHO | World malaria report 2018). After being inoculated in the skin of a host during the bite of an infected Anopheles mosquito, the sporozoite form of the parasite is quickly conveyed by the blood to the liver. Once there, it is sequestered via interactions with heparan sulphate proteoglycans (HSPGs), crosses the 
endothelial barrier, and then migrates through several parenchymal cells before invading a hepatocyte by forming a membrane-bound compartment called the parasitophorous vacuole membrane (PVM), where it can develop and multiply to form merozoites that initiate the pathogenic erythrocytic cycle (Mota et al., 2001).

Sporozoite entry into hepatocytes is a highly complex process that involves coordinated interaction of sporozoite and host factors for initiation of infection, which may offer a multitude of promising therapeutic targets (Dundas et al., 2019). Currently, two major host cell surface proteins are well known to be involved in sporozoite invasion, the tetraspanin CD81 and the scavenger receptor class B type1 (SR-BI) (Silvie et al., 2003; Rodrigues et al., 2008; Yalaoui et al., 2008a), but how they interact with specific parasite ligands remains largely unknown (Silvie et al., 2007). Moreover, the molecular events involved in sporozoite invasion are further complicated because they depend on the plasmodial species and the host cell type (Risco-Castillo et al., 2014). Indeed, CD81 is essential for P. falciparum and $P$. yoelii sporozoite invasion (Silvie et al., 2003) but is not required by $P$. vivax, which depends only on SR-BI. On the other hand, SR-BI is not required for P. falciparum and P. yoelii infections (Manzoni et al., 2017). However, P. berghei sporozoites can enter cells alternatively via either CD81 or SR-BI (Manzoni et al., 2017). The choice of route by which sporozoites invade hepatocytes has been shown to be determined by the parasite 6-cysteine domain protein P36 in a species-specific manner (Manzoni et al., 2017). In addition, SR-BI has been found to be essential for subsequent parasite development inside hepatocytes but not CD81 (Silvie et al., 2003; Rodrigues et al., 2008). Most recently, another host factor, aquaporin 3 (AQP3), which is a water and glycerol channel belonging to the aquaglyceroporin subfamily, has been implicated in the maturation of parasites after invasion (Posfai et al., 2018; Posfai et al., 2020).

Previous studies have shown that the human hepatocarcinoma HepG2 cells express SR-BI but not CD81, and as a consequence, they support infection by $P$. berghei but not by P. falciparum or P. yoelii sporozoites (Silvie et al., 2006b; Manzoni et al., 2017). Interestingly, ectopic expression of CD81 in HepG2 cells is sufficient to confer susceptibility to $P$. yoelii but not to $P$. falciparum sporozoites (Silvie et al., 2006b), implying the existence of additional host factors that are critical for $P$. falciparum infection.

The aim of this study was to identify host proteins required for successful infection of human hepatocytes by $P$. falciparum sporozoites. The approach adopted was that of a differential transcriptome analysis of naturally permissive human hepatocytes versus non-permissive hepatoma HepG2-CD81 cells. Among the most downregulated genes in the nonpermissive cells, we focused on that coding for aquaporin-9 (AQP9), a water and glycerol channel previously shown to be involved in mice susceptibility to $P$. berghei blood stage infection (Liu et al., 2007). The contribution of AQP9 to the Plasmodium liver stage of $P$. falciparum, $P$. yoelii and $P$. berghei was investigated using primary human hepatocytes, human hepatoma cells and primary murine hepatocytes.

\section{MATERIALS AND METHODS}

\section{Ethics Statement}

Human hepatocytes were isolated from liver segments taken after oral informed consent was obtained from adult patients undergoing partial hepatectomy as part of their medical treatment (Service de Chirurgie Digestive, Hépato-BilioPancréatique et Transplantation Hépatique, Hôpital PitiéSalpêtrière, Paris, France). The collection and use of this material for the purposes of the study presented here were undertaken in accordance with French national ethical guidelines under Article L. 1121-1 of the "Code de la Santé Publique". Given that the tissue samples were classed as surgical waste, they were used anonymously. Moreover, because they were not in any way genetically manipulated, article L. 1211-2 stipulates that their use for research purposes is allowed provided that the patient does not express any opposition to this to the surgeon prior to surgery after being informed of the nature of the research in which they might be potentially employed. The collection and use of this material were furthermore approved by the Institutional Review Board (Comite de Protection des Personnes) of the Centre Hospitalo-Universitaire PitiéSalpêtrière, Assistance Publique-Hôpitaux de Paris, France. All animal care and procedures were carried out in strict accordance with the recommendations of the Guide for the Care and Use of Laboratory Animals of the European Union "European directive $86 / 609 / E E C$ ". The protocol was approved by the Ethics Committee for Animal Experiments of University Pierre et Marie Curie, Paris 6, France (Permit Number: 75-1087).

\section{RNA Isolation, Microarray Protocol and Differential Analysis of Transcriptomes}

Three sets of human primary hepatocytes (Supplementary Table 1) were cultured for one week in a differentiation maintenance medium (complete medium for hepatocyte culture supplemented with $2 \%$ DMSO) (Isom et al., 1985). Hepatoma-derived HepG2-CD81 cells (Silvie et al., 2006b) from three different cultures were grown to subconfluence in $75 \mathrm{~cm}^{2}$ flasks. Cells were lysed with TRIzol (Invitrogen, Life Technologies), and RNA was purified according to the manufacturer's instructions. RNA was equally purified from $1 \mathrm{~g}$ of human liver for use as a reference in all the microarray hybridizations. RNA concentration and purity were assessed using a NanoDropND-1000 Spectrophotometer (Thermo Scientific) and the Agilent 2100 Bioanalyser.

Transcriptomes of $P$. falciparum permissive human hepatocytes (HHs) and refractory HepG2-CD81 hepatoma cells (HCs) were analysed using a human 1A oligoarray kit (pangenomic human 22K DNA microarrays from Agilent Technologies, Les Ulis, France). Cyanine-labelled cDNA probes were synthesized from $20 \mu \mathrm{g}$ of $\mathrm{HH}, \mathrm{HC}$, or total liver RNA using a CyScribe Post-Labelling kit (GE Healthcare Life Sciences, Vélizy, France). cDNA probes synthesized from cell RNAs were labelled with cyanine 3 (Cy3), while probes synthesized from the liver reference RNA were labelled with cyanine 5 (Cy5). Cy3- and Cy5-labelled probes were then co-hybridized to spots 
on the same microarray. Signals were extracted using a Genepix 4000B scanner (Axon Instruments) and Genepix Pro 6.0 software and normalized with VARAN software (http://www. bionet.espci.fr/varan/), allowing calculation of the $\mathrm{HH} / \mathrm{HC}$ intensity ratio for each targeted gene spotted on the microarray as the ratio of $(\mathrm{HH} /$ reference intensity ratio) versus (HC/reference intensity ratio). All microarray procedures were performed in accordance with the manufacturer's instructions. The CD81 synthetic oligonucleotide probe used here is complementary to the endogenous 5 -untranslated region of wild type $C D 81$ but not to the ectopic $C D 81$ expressed by the HepG2-CD81 cell line, therefore the probe recognizes only endogenous CD81 mRNA.

\section{Hepatocytes, Hepatoma Cells and Cell Culture}

Primary human hepatocytes were isolated from healthy parts of the liver fragments as previously described (Yalaoui et al., 2008a). Primary mouse hepatocytes were isolated as previously described (Yalaoui et al., 2008a) from $A Q P 9^{-/-}$mice backcrossed onto a C57BL/6J background and their $A Q P 9^{+/+}$littermate controls aged 7 to 10 weeks and gender matched (Rojek et al., 2007). These mice were kindly provided by Pr. S. Nielsen (Aarhus University, Aarhus, Denmark) and Pr. M. Amiry-Moghaddam (University of Oslo, Oslo, Norway). Human and mouse hepatocytes were seeded at a density of $8 \times 10^{4}$ cells and $4 \times$ $10^{4}$ cells, respectively, in 96-well microplates precoated with rat tail collagen I (Becton Dickinson) and cultured at $37^{\circ} \mathrm{C}$ in $5 \%$ $\mathrm{CO}_{2}$ in William's E medium (Gibco, Life Technologies, Saint Aubin, France) supplemented with $2 \%$ penicillin-streptomycin, $1 \%$ sodium pyruvate, $1 \%$ L-glutamine, $1 \%$ insulin-transferrinselenium, $1 \%$ non-essential amino acids (solutions for cell culture, Gibco) and 10\% foetal bovine serum (FBS, Gibco). For human hepatocyte, the culture medium was also supplemented with $10^{-7} \mathrm{M}$ dexamethasone (Sigma) and 2\% DMSO. HepG2CD81 (Bartosch et al., 2003; Silvie et al., 2006b) human hepatoma cells were cultured in DMEM (Gibco) supplemented with $10 \%$ FBS, $1 \%$ glutamine and $1 \%$ penicillin-streptomycin (Gibco). HepaRG human hepatoma cells, a gift from Pr. C. Guillouzo (INSERM, Rennes, France), were cultured in William's E medium (Gibco) supplemented with $1 \%$ penicillinstreptomycin, $1 \%$ L-glutamine, insulin $(5 \mu \mathrm{g} / \mathrm{ml}), 5 \times 10^{-5} \mathrm{M}$ hydrocortisone and 10\% FBS (Thermo Scientific, Villebon-surYvette, France). Mouse and human hepatocytes as well as human hepatoma cells were cultured for at least 24 hours before further studies.

\section{Plasmodium Parasites and In Vitro Infection}

P. yoelii (265BY strain), P. berghei (PbGFPCON, ANKA strain) (Franke-Fayard et al., 2004) and P. falciparum (NF54 strain) sporozoites were obtained by dissection of salivary glands from infected Anopheles stephensi mosquitoes bred and infected in the insectary facilities of UMRS 945 (Paris, France) for rodent Plasmodium-infected mosquitoes and the Department of Medical Microbiology, Radboud University (Nijmegen,
Holland) for P. falciparum-infected mosquitoes. Infection assays were performed in 96-well plates. Cells were inoculated with $3 \times 10^{4} \mathrm{P}$. falciparum or $2 \times 10^{4} \mathrm{P}$. berghei or $P$. yoelii Plasmodium sporozoites per well and then centrifuged for $10 \mathrm{~min}$ at $2000 \mathrm{rpm}$ to allow fast parasite sedimentation onto the target cells. After 3 hours at $37^{\circ} \mathrm{C} / 5 \% \mathrm{CO}_{2}$, thus allowing sporozoite penetration into hepatocytes, cultures were washed and further incubated in fresh medium for 2 or 4 days (for $P$. yoelii and $P$. berghei or $P$. falciparum, respectively) until quantification of EEFs or directly fixed in PFA for quantification of parasite entry.

\section{Gene Knockdown Using Small Interfering RNAs}

For gene knockdown, we used small double-stranded RNA oligonucleotides (siRNAs) targeting human CD81 (sihCD81; 5'- GCA CCA AGT GCA TCA AGT A -3'), human AQP3 (AQP3_5 siRNA from Qiagen HP GenomeWide), human AQP9 (sihAQP9 ${ }^{1}$; 5'-CTG CTG ATC GTG GGA GAA A-3' and sihAQP9s ${ }^{2}$ to 5 corresponding respectively to siRNAs from Qiagen HP GenomeWide - AQP9_1 HP siRNA; AQP9_2 HP siRNA; AQP9_4 HP siRNA; AQP9_5 HP siRNA), and hCD92 (sihCD92; 5'- AAG GCA AGA ACT GAA AAC T -3'), used as a negative control. Primary hepatocytes were transfected with 30 nM siRNA in 96-well microplates using Lipofectamine RNAi MAX (Invitrogen) according to the manufacturer's recommendations. HepaRG cells $\left(5 \times 10^{6}\right.$ cells in $400 \mu \mathrm{l}$ of RPMI) were transfected with 200 pmol of siRNA via electroporation (300 V, $500 \mu \mathrm{F})$ (Silvie et al., 2006a) using a Gene Pulser apparatus (Bio-Rad,Ivry, France). siRNAtransfected hepatoma cells or hepatocytes were then cultured for 48 hours before mRNA expression analysis or for 72 hours before protein expression or sporozoite infection efficiency analysis.

\section{Lentiviral Vector Constructs and Cell Transduction}

The lentiviral constructs pTRIP-CMV-hAQP9 $\Delta \mathrm{U} 3$ and pTRIP$\mathrm{CMV}-\mathrm{hCD} 81 \Delta \mathrm{U} 3$ were generated via gateway recombination cloning (Invitrogen). Briefly, hAQP9 and hCD81 coding sequences were PCR amplified and cloned into a pENTR/D/ TOPO plasmid (Invitrogen) to generate entry clones. LR clonase II recombination was performed using the entry clones and the pTRIP-CMV-rfa Gateway $\Delta \mathrm{U} 3$ destination vector as described previously (Russ et al., 2008). The pTRIP-CMV-GFP $\Delta$ U3 vector (Castaing et al., 2005) was used as a control. Lentiviral vector stocks were produced by transient transfection of 293T cells with the p8.91 encapsidation plasmid (Zufferey et al., 1997), the VSV glycoprotein-G encoding pHCMV-G plasmid (Yee et al., 1994), and the pTrip $\Delta \mathrm{U} 3$ lentiviral vector as previously described (Zennou et al., 2000). Supernatants were treated with DNAse I (Roche Diagnostics) prior to ultracentrifugation, and the resulting pellet was resuspended in PBS, separated into aliquots and frozen at $80^{\circ} \mathrm{C}$ until use. Primary human hepatocytes and hepatoma cells were transduced through addition of a 1:1000 concentrated lentiviral preparation. After an incubation period 
of three hours, the cells were washed. Three days later, the cultures were processed for protein expression analysis as described in western blotting procedures or infected with sporozoites and cultured for four days until exo-erythrocytic forms (EEFs) quantification to evaluate hepatocyte permissiveness to Plasmodium infection.

\section{Quantification of hAQP9 and hCD81 Transcripts via Real-Time PCR}

RNA extractions were performed with a PureLink RNA Mini Kit (Ambion, Life Technologies) according to the manufacturer's recommendations. DNase treatment (PureLink DNase Set; Ambion, Life Technologies) was performed during the extraction. The quality and purity of RNAs were assessed with the Agilent 2100 Bioanalyser. Reverse transcription was realized with a SuperScript VILO cDNA Synthesis Kit (Invitrogen) according to the manufacturer's instructions, using $1 \mu \mathrm{g}$ of RNA in a $20 \mu \mathrm{L}$ final volume and duplicates for all samples. Quantitative real-time PCRs (qPCRs) were carried out using the SYBR Green method (SYBR GreenER qPCR SuperMix Universal, Invitrogen) with a MX3005P qPCR System (Agilent Technologies). The volume of cDNA used was equivalent to 100 ng of RNA per reaction, and the primers were used at a final concentration of $200 \mathrm{nM}$. Primer pairs based on the cDNA sequence were specifically designed for hAQP9 (5' ttgcaacatacccagctccgtatc-3' and 5'-accaaagggcccactacaggaat-3') and for hCD81 (5'-.tcatcctgtttgcctgtgaggt-3' and 5' tgaggtggtcaaagcagtcagt- $\left.3^{\prime}\right)$. All samples were run in duplicate with the two different cDNAs synthesized, and gene expression data were normalized according to the level of TBP (TATA boxbinding protein) (Ceelen et al., 2011).

\section{Protein Expression Analysis via Western Blotting and Cell Immunofluorescence}

Primary human hepatocytes were lysed at $4^{\circ} \mathrm{C}$ for 30 minutes in $30 \mathrm{mM}$ Tris ( $\mathrm{pH} 7.4$ ), $150 \mathrm{mM} \mathrm{NaCl}, 0.02 \% \mathrm{NaN}_{3}$, protease inhibitors (PMSF, INH) and 1\% Triton X-100. CD81 and AQP9 expression was analysed via western blotting using a mouse antihCD81 mAb (1:1000) and a mouse anti-m/hAQP9 mAb (G3, Santa Cruz) (1:100), respectively, followed by a goat anti-mouse Alexa Fluor 680 conjugate (Molecular Probes, Life Technologies) (1:15000). Monoclonal mouse anti-tubulin (Abcam) (1:5000) was used as a standard. Data were acquired and quantified with an Odyssey Infrared Imaging System (LI-COR Biosciences, Lincoln, NE).

Quantification of hCD81 and AQP9 expression at the surface of human hepatocytes was performed as previously described for murine hepatocytes (Yalaoui et al., 2008a). Cells were washed with PBS, and immunolabelled for human AQP9 using a goat anti-hAQP9 (C18, Santa Cruz) antibody and a donkey anti-goat IgG-Alexa 488 conjugate antibody (Molecular Probes). CD81 was labelled with a mouse anti-hCD81 antibody (TS81, provided by Dr. E. Rubinstein, INSERM, Villejuif, France) and revealed with donkey anti-mouse IgG-Alexa 568 (Molecular Probes). Cell density was evaluated with diamidino-phenyl-indole (DAPI, Sigma) and used to normalize CD81 expression. Fluorescence intensity was acquired with a Flexstation Universal Microplate Reader (Molecular Devices, St Grégoire, France).

\section{Quantification of Parasite Infection or Invasion via Immunofluorescence}

After fixation of cultures with cold methanol, EEFs were identified using anti-HSP70 serum, prepared from mice immunized with a recombinant HSP70 protein (a gift from Pr. D. Mattei, Institut Pasteur, Paris, France), stained with an Alexa 488-conjugated or Alexa Fluor 680-conjugated goat anti-mouse immunoglobulin (Molecular Probes) and counted under a fluorescence microscope or using the Odyssey Infrared Imaging System as described previously (Gego et al., 2006). Nuclei were stained with $1 \mu \mathrm{g} / \mathrm{ml}$ DAPI. Quantification of parasite entry was carried out using double immunostaining with the appropriate mouse monoclonal anti-CSP antibody for P. falciparum $\operatorname{CSP}^{P \text {. falciparum }}$ and $P$. yoelii $\left(\mathrm{CSP}^{P .}\right.$ yoelii $)$ (Dr. Y. Charoenvit, Naval Medical Research Center; Silver Spring, MD), or a TRAP antibody for $P$. berghei (Dr. A. Crisanti, Imperial College, London, UK), thus allowing discrimination of intracellular and extracellular sporozoites, as previously described (Rénia et al., 1988; Silvie et al., 2002).

\section{Sporozoite Cell Traversal Assay}

Hepatocytic cell traversal was analysed using a dextran incorporation FACS assay (Prudêncio et al., 2008). Immediately after electroporation in the presence of siAQP9, siCD81, or siCD92, HepaRG cells were plated and cultivated in a $25 \mathrm{~cm}^{2}$ culture flask at $37^{\circ} \mathrm{C} / 5 \% \mathrm{CO}_{2}$. Forty-eight hours later, the cells were trypsinized for direct mRNA expression analysis or seeded in 48 -well plates ( $8 \times 10^{4}$ cells/well) for a cell traversal assay. After 24 hours, they were incubated with $10^{5} \mathrm{P}$. yoelii or $P$. berghei sporozoites for 2 hours or with $10^{5} \mathrm{P}$. falciparum sporozoites for 3 hours in the presence of rhodamine-dextran lysine fixable (10000 MW, Molecular Probes). After washing, the cells were trypsinized, fixed with $1 \%$ glutaraldehyde and analysed via FACS using an LSR Fortessa flow cytometer (BD Biosciences); $10^{4}$ cells were analysed (van Schaijk et al., 2008).

\section{Infection Inhibition Assay With the AQP9 Inhibitor Phloretin}

To assess the function of AQP9 in Plasmodium parasite infection, we tested the effect of phloretin (Sigma, Saint Quentin Fallavier, France), an inhibitor that restricts the solute permeability of AQP9 (Tsukaguchi et al., 1998; Yang and Verkman, 2002; Liu et al., 2007), on infection of human primary hepatocytes by $P$. falciparum. Phloretin was coincubated with the sporozoites in the first 2 hours of the invasion step. Thereafter, the cells were incubated with fresh complete medium for three additional days until EEF quantification. Phloretin concentrations up to $500 \mu \mathrm{M}$ were tested because this concentration has previously been shown to inhibit $60 \%$ of urea and glycerol transport (Tsukaguchi et al., 1998) and to not affect rat hepatocyte viability (Mosmann, 1983; Huebert et al., 2002). Using an MTT toxicity assay (Mosmann, 1983), we assessed that phloretin was not toxic to primary 
human hepatocytes under the assay conditions (Supplementary Figure 1). We also assessed that phloretin was not toxic on Plasmodium sporozoites preincubated with different concentrations of phloretin (Supplementary Figure 2).

\section{Data Analysis}

GraphPad Prism 7 statistical Software (GraphPad. Software, San Diego, CA, USA) was used for data analysis and graphing. All values were expressed as means and standard deviations (SD). A $p$-value of 0.05 or less was considered as statistically significant.

\section{RESULTS}

\section{Comparative Transcriptomics of Plasmodium falciparum Permissive and Non-Permissive Hepatocytes Identified AQP9 as a Candidate Host Protein Involved in Sporozoite Invasion}

To identify novel host factors and further understand human hepatocyte susceptibility to $P$. falciparum sporozoite invasion, we performed differential transcriptomic analysis of naturally $P$. falciparum-permissive human hepatocytes ( $\mathrm{HHs}$ ) and refractory human hepatoma HepG2-CD81 cells (HCs) using microarray technology. Only genes downregulated in nonpermissive hepatoma cells were taken into account. Our results showed that a total of 276 genes were downregulated in nonpermissive hepatoma cells compared to the permissive hepatocytes, with $\mathrm{HH} / \mathrm{HC}$ ratios higher than 3. A list of the top 30 downregulated genes with the highest ratios is presented in (Supplementary Table 2). As expected, CD81 (with an HH/ HC ratio of 14.7) was found to be among the most downregulated genes in hepatoma cells (Figure 1A and Supplementary Figure 3A). It should be noted that the CD81 cDNA probe used on the DNA chips was designed to detect only endogenous CD81 mRNA but not ectopic CD81. SR-BI (HH/ $\mathrm{HC}=6$ ) was also found amid the downregulated genes in hepatoma cells (Figure 1A and Supplementary Figure 3A). In contrast, the expression ratio of the irrelevant CD9 gene $(\mathrm{HH} /$ $\mathrm{HC}=1.06$ ), which is known as not involved in hepatocyte permissiveness to Plasmodium infection (Yalaoui et al., 2008b), was not changed (Figure 1A and Supplementary Figure 3A).

From evaluation of each candidate gene, $A Q P 9$ which belongs to the aquaglyceroporin subfamily, a transmembrane channel protein that conducts water and glycerol (Liu et al., 2007), was selected for functional investigations, because it was among the most downregulated genes in the non-permissive HepG2 cells (Figure 1A and Supplementary Figure 3A), and evidence from a previous study showed it to be involved in mouse susceptibility to P. berghei blood stage infection (Liu et al., 2007). Interestingly, another aquaglyceroporin, $A Q P 3$, which was reported to be involved in $P$. berghei development within human hepatoma cells and in $P$. vivax development within human primary hepatocytes but is not essential for sporozoite invasion (Posfai et al., 2018; Posfai et al., 2020), was not found to be significantly differentially expressed between the two cell types $(\mathrm{HH} / \mathrm{HC}$ ratio

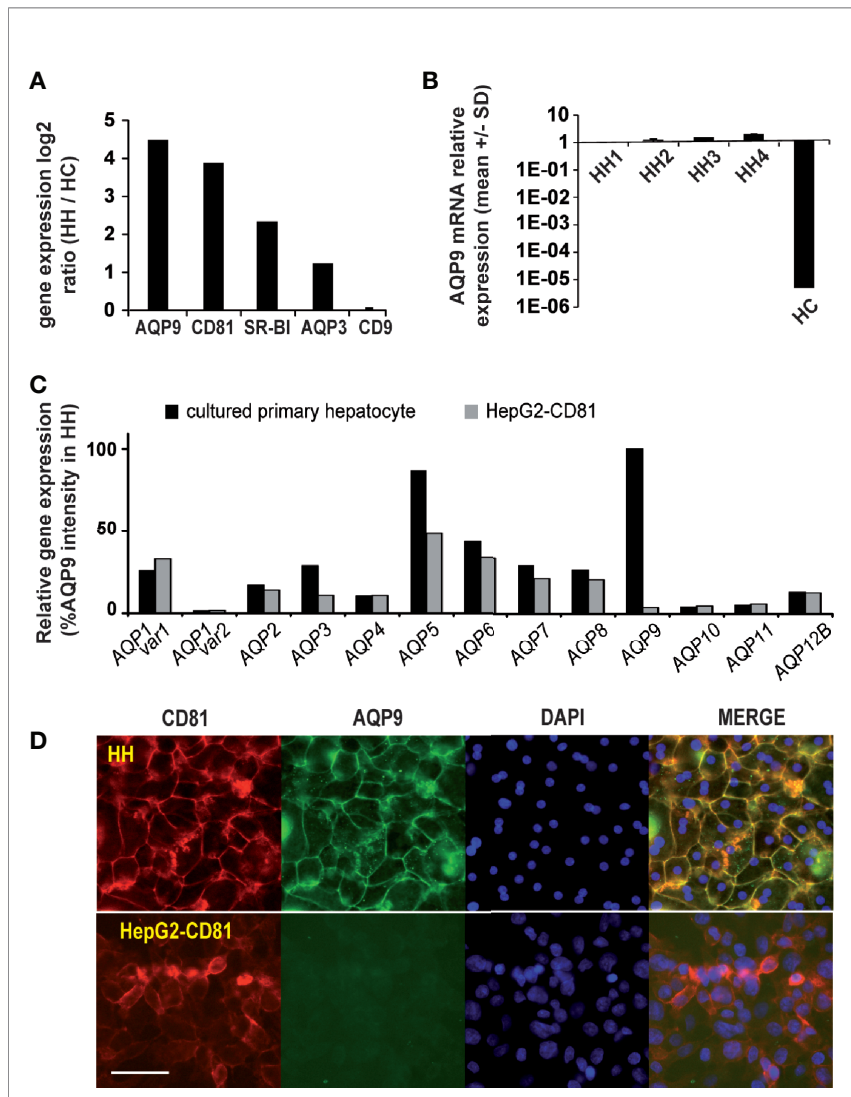

FIGURE 1 | AQP9 and CD81 are expressed at higher levels in $P$. falciparum permissive human hepatocytes than in refractory hepatoma cells. (A) Transcriptomes of human hepatocytes (HHs) and human hepatoma HepG2CD81 cells (HCs) were analysed using a 22K pan-genomic human oligo microarray (Agilent Technologies). AQP9, CD81, AQP3 and CD9 gene expression levels are presented as average log2 gene ratios in $\mathrm{HH} / \mathrm{HC}$. (B) RT-qPCR analysis of AQP9 mRNA expression in 4 sets of $\mathrm{HHs}$ and in HCs. Variations in transcript levels were calculated after normalisation to expression of the TBP gene and are presented relative to the AQP9 mRNA level in $\mathrm{HH} 1$. (C) Relative gene expression of aquaporins 1 to $12 \mathrm{~B}$ in $\mathrm{HHs}$ from 3 donors and $\mathrm{HCs}$ is presented as the mean \% of the AQP9 gene expression level in $\mathrm{HHs}$, determined with the $22 \mathrm{~K}$ pan-genomic human oligo microarray. (D) Immunofluorescence analysis of CD81 and AQP9 expression on HHs (upper panel) and HCs (lower panel). Cells were fixed, permeabilized, and incubated with a mixture containing a mouse monoclonal antibody against human CD81 and human AQP9 antibody and reacted with the appropriate secondary antibodies conjugated to Alexa 568 and Alexa 488, respectively. The white scale bar represents $100 \mu \mathrm{m}$.

of 2.3) (Figure 1A). The relatively high AQP9 gene expression in primary hepatocytes was further confirmed in hepatocytes isolated from 4 additional donors using qPCR (Figure 1B; Supplementary Table 1). The expression of other aquaporin genes was not or was only moderately downregulated (Figure 1C and Supplementary Figure 3B). Moreover, immunofluorescence experiments showed that the drastic downregulation of the AQP9 transcript in HepG2CD81 cells was accompanied by a near total lack of protein expression (Figure 1D). Overall, as a membrane-bound protein highly expressed in infection-permissive cells and almost silent in non-permissive cells (Figure 1D and Supplementary Figure 4), 

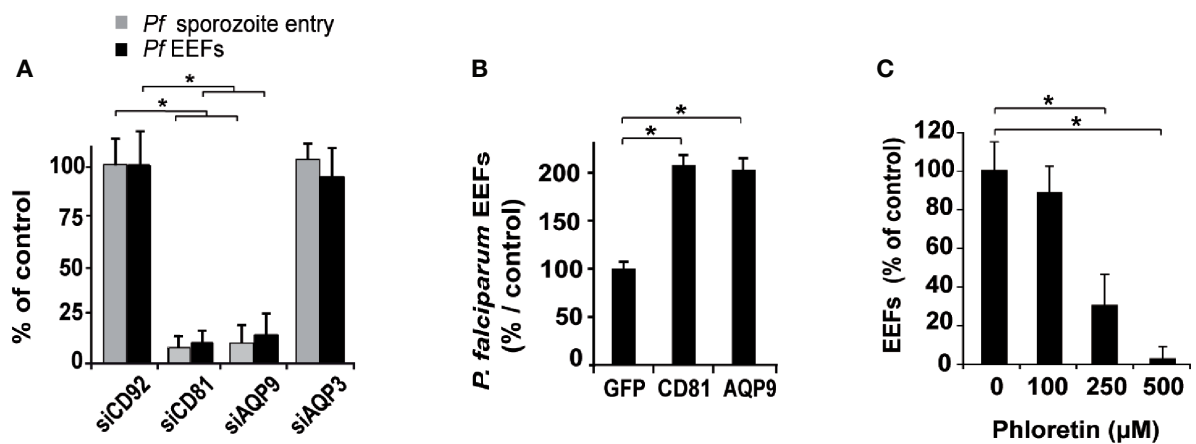

FIGURE 2 | Human hepatocyte permissiveness to $P$. falciparum sporozoite infection depends on AQP9 expression. (A) Hepatocytes were transfected with sihRNAs and tested for their permissiveness to $P$. falciparum sporozoite infection: sihCD92-, sihCD81-, sihAQP91- and sihAQP3-transfected human hepatocytes were infected three days after transfection with $P$. falciparum sporozoites and cultured for 3 hours to evaluate sporozoite entry via double immunostaining for PfCSP protein (grey bars) or for four days to evaluate cell infection after immunolabelling of EEFs using HSP70 antibody (black bars). Sporozoite and EEF numbers are expressed as a percentage of those in sihCD92-transfected control hepatocytes. ${ }^{\star} p=0.0006$. (B) Hepatocytes overexpressing CD81 or AQP9 were tested for their permissiveness to $P$. falciparum infection: hepatocytes transduced with a lentiviral vector expressing GFP protein, as a control, hCD81, or hAQP9 were infected three days later with sporozoites and cultured for four days until EEF quantification. EEF numbers are expressed as a percentage of those in GFP-transduced control hepatocytes. The mean schizont numbers per well in controls of three independent experiments were 141, 203, and 314. * $p=0.05$. (C) Hepatocytes were treated for the first two hours following sporozoite inoculation with three doses of phloretin. Hepatocytes were then washed and incubated with fresh complete medium for three additional days until EEF quantification. EEF rates are relative to those in untreated hepatocytes. ${ }^{*} p=0.0009$. All analyses were carried out in triplicate wells, and the results are expressed as the mean \pm standard deviation. $p$ values were determined by Mann-Whitney $U$ test.

hepatocyte AQP9 is a candidate protein that could be involved in sporozoite entry into cells.

\section{Aquaporin-9 Is Required for Efficient P. falciparum Sporozoite Entry Into Human Hepatocytes}

To investigate the potential role of AQP9 in human hepatocyte infection by $P$. falciparum sporozoites, we performed $A Q P 9$ gene silencing using five smalls interfering RNAs (siRNAs). These siRNAs were first tested for their ability to ablate $A Q P 9$ gene expression in hepatocytes (Supplementary Figure 5A). sihCD92 and sihCD81 were used as negative and positive controls, respectively. Gene silencing procedure as well as sihCD92 which are routinely used in our laboratory have no impact on Plasmodium infection. Next, using western blotting and quantitative immunofluorescence, we confirmed that siAQP9 does not affect CD81 protein levels (Supplementary Figures 5A, B). Microscopic observation of the cultures transfected with either sihAQP9, sihCD81 or sihCD92 showed no obvious morphological alteration of the cells nor of the cell layer integrity (Supplementary Figure 6). Finally, we checked the efficiency of the five siRNAs in blocking sporozoite infection of hepatocytes (Supplementary Figure 5C). Silencing with sihAQP9 ${ }^{1,3,5}$ resulted in an $\sim 70 \%$ reduction in hepatocyte permissiveness to $P$. falciparum compared to the control sihCD92-treated hepatocytes. This reduction was similar to that obtained with sihCD81. sihAQP9 4 caused an $\sim 35 \%$ reduction in hepatocyte permissiveness, while sihAQP9 ${ }^{2}$ had no effect. The reduction in hepatocyte infectivity observed with sihAQP9s was proportional to the inhibition of $A Q P 9$ expression. Together, these results show that AQP9 and CD81 are individually required for Plasmodium infection but that neither is able to compensate for silencing of the other. We choose siAQP9 ${ }^{1}$ among the 3 siRNA
(siAQP9 ${ }^{1,3}$ and ${ }^{5}$ ) showing a potent and comparable silencing of AQP9 protein to be used for the rest of our experiments.

To further define the role of AQP9, we investigated its specific contribution to sporozoite entry into hepatocytes. Hepatocytes were inoculated with $P$. falciparum sporozoites three days after transfection, and their entry was evaluated by double immunostaining three hours post-sporozoite infection (Rénia et al., 1988; Silvie et al., 2002). The entry of parasites into sihAQP9- and sihCD81-treated hepatocytes was decreased by $90 \%$ and $92 \%$, respectively, compared to sihCD92-treated cells (Figure 2A and Supplementary Figure 7A), and this inhibitory effect was similar to that observed for infection efficiency. In contrast, $A Q P 3$ gene silencing did not affect Plasmodium sporozoite infection or entry (Figure 2A and Supplementary Figure 7A), corroborating results obtained elsewhere (Posfai et al., 2018).

We then examined whether AQP9 overexpression might improve cell permissiveness to $P$. falciparum sporozoite infection, as overexpression of CD81 in hepatocytic cell lines did for rodent Plasmodium species (Silvie et al., 2006b). To this end, we transduced human hepatocytes that naturally express endogenous $C D 81$ and $A Q P 9$ with a lentiviral vector allowing expression of $C D 81$ or $A Q P 9$, and then cultured the hepatocytes for three days prior to Plasmodium sporozoite infection. Cells transduced with a vector encoding the irrelevant GFP protein were used as a control (GFP expression in liver cells was previously reported to not alter Plasmodium infection efficiency (Yalaoui et al., 2008b)). Overexpression of AQP9 (as assessed by western blotting, Supplementary Figure 8) induced a two-fold increase in hepatocyte permissiveness to $P$. falciparum infection compared with GFP-transduced hepatocytes, comparable to that of CD81 (Figure 2B). Pharmacologic inhibition of AQP9 by phloretin during the first two hours of 

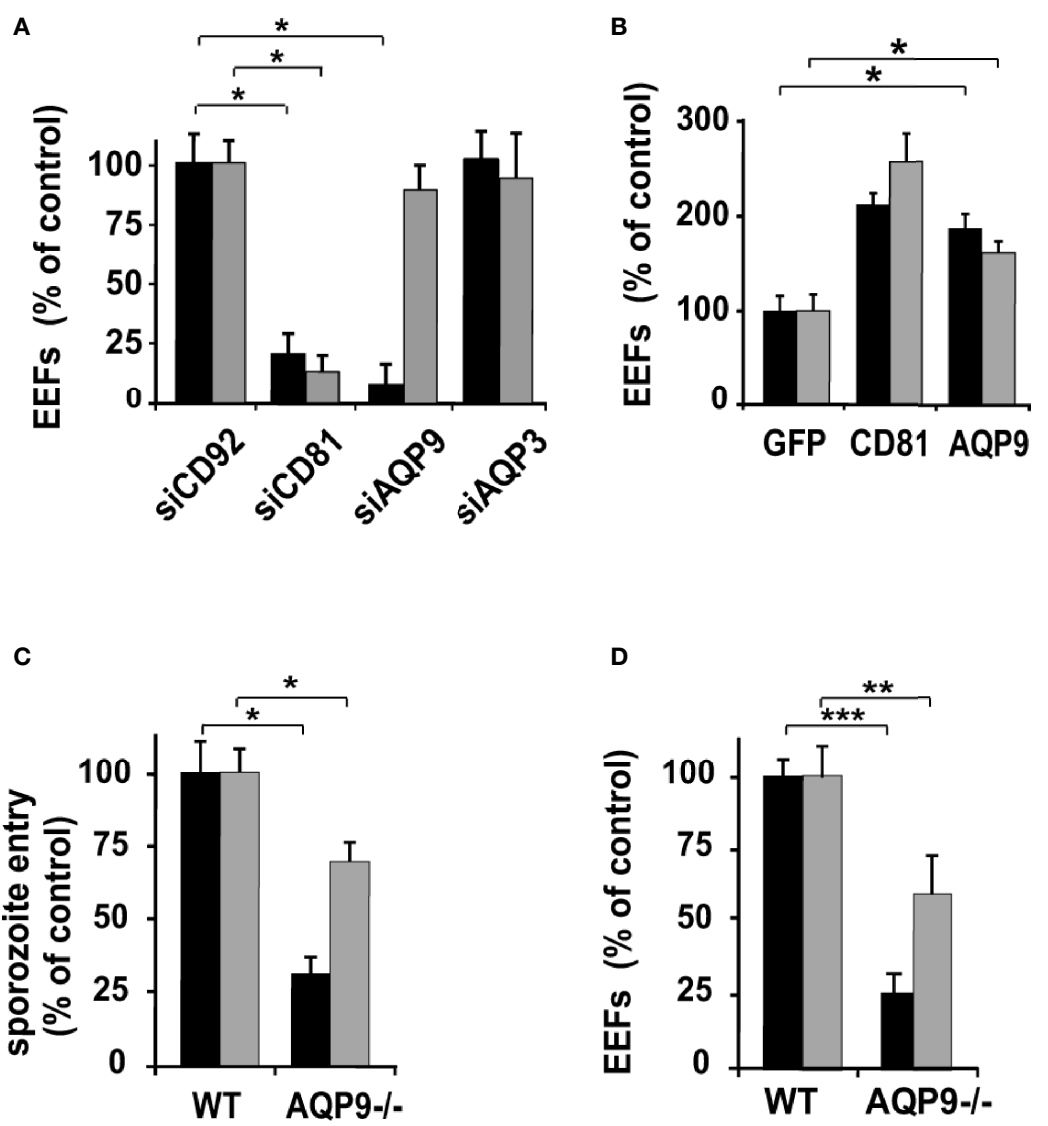

\section{- P. berghei $\quad \square$ P. yoelii}

FIGURE 3 | Hepatocyte permissiveness to rodent parasites differentially depends on AQP9 according to parasite/host cell combinations. (A) sihRNA-transfected human hepatocytes (HHs) were infected with sporozoites three days post-transfection and cultured for two days until EEF quantification. EEF values are expressed as a percentage of those in control sihCD92-transfected hepatocytes. ${ }^{*} p=0.0001$. (B) Three days post lentiviral transduction, HHs overexpressing GFP protein, hCD81, or hAQP9 were cultured for two days until EEF quantification. EEF numbers are expressed as a percentage of those in control GFP-transduced hepatocytes. ${ }^{*} p=0.0079$. (C, D) Primary hepatocytes isolated from wild-type (WT) and AQP9 knockout (AQP9-/-) mice were infected with P. berghei or P. yoelii. Sporozoite cell entry was evaluated $3 \mathrm{~h}$ post-infection using double immunostaining (C), whereas EEF quantification was performed after $48 \mathrm{~h}$ of culture (D). Penetration rates and EEF numbers are expressed as a percentage of those in control WT hepatocytes. ${ }^{\star} p=0.05 ;{ }^{* *} p=0.0018 ;{ }^{* \star *} p=0.0004$. The results are expressed as the mean \pm standard deviation. $p$ values were determined by Mann-Whitney $U$ test.

hepatocyte co-incubation with sporozoites resulted in a significant reduction in hepatocyte infection in a dosedependent manner (Figure 2C). We also found that cell traversal of $P$. falciparum sporozoites was not affected by either CD81 or AQP9 silencing (Supplementary Figure 9A).

Since $A Q P 9$ RNA silencing induced a major, but incomplete, inhibition of $P$. falciparum infection, with 10 to $30 \%$ residual development of liver schizonts, we could assess whether AQP9 might play a role in the maturation of the parasite. SihAQP9 ${ }^{1}$ treated human hepatocytes infected by $P$. falciparum sporozoites were labelled four days post-inoculation with an anti-PfHSP70 antibody in order to measure the size of the schizonts.
Fluorescence microscopy analysis revealed that schizont diameters were similar in sihCD92, sihCD81 and sihAQP9 ${ }^{1}$ treated hepatocytes (Supplementary Figure 10A).

Taken together, these data clearly provide evidence that $A Q P 9$ expression is important for P. falciparum sporozoite entry into human hepatocytes but have no influence on parasite development at the liver stage. Interestingly, the magnitude of the effect of $A Q P 9$ silencing in human hepatocytes on infection by $P$. falciparum, either on parasite entry or on the number of schizonts, is comparable to that of CD81 silencing. Similarly, both AQP9 overexpression and CD81 overexpression significantly enhance $P$. falciparum infection of human hepatocytes. 


\section{The Rodent Parasites P. yoelii and $P$. berghei Differentially Depend on AQP9 for Infection of Liver Cells}

We further extended our experiments to study liver infection by the rodent malaria parasites $P$. yoelii and $P$. berghei. Given that the requirement for host proteins that confer permissiveness to Plasmodium infection may differ according to the species of the target cell (Silvie et al., 2007) and that P. yoelii and P. berghei sporozoites are capable of infecting human hepatocytes in vitro (Silvie et al., 2006b), the role of AQP9 was investigated in infection of human hepatocytes by these rodent Plasmodium species. The permissiveness of sihCD81- and sihAQP9 ${ }^{1}$-treated human hepatocytes to $P$. berghei infection was reduced by $79 \%$ and $\sim 92 \%$, respectively. As expected, silencing of CD81 reduced P. yoelii infection by $\sim 87 \%$, but surprisingly, silencing of $A Q P 9$ did not affect $P$. yoelii infection (Figure $\mathbf{3 A}$ and Supplementary Figure 7B). In fact, the dramatic effect of $A Q P 9$ silencing on infection by $P$. berghei was also somewhat unexpected, because it is well known that human HepG2 hepatoma cells can be readily infected by this parasite, despite undetectable AQP 9 expression. This paradox was further investigated in HepaRG cells that express AQP9. P. berghei infection of these cells was markedly decreased by $A Q P 9$ RNA silencing (Supplementary Figures 11A, B), which suggests that this parasite uses AQP9dependent pathways to invade human liver cells naturally expressing $A Q P 9$ but is able to use alternative pathways in hepatoma cells that do not express AQP9 (see Table 1). CD81 or $A Q P 9$ overexpression in human hepatocytes transduced with CD81- or AQP9-expressing lentiviral vectors led to an increase in the number of liver schizonts in each rodent species (Figure 3B). Finally, the availability of AQP9 knockout mice prompted us to test permissiveness of primary hepatocytes isolated from wild-type or AQP9 knockout mice to rodent Plasmodium sporozoites. The total lack of AQP9 resulted in a reduction in hepatocyte permissiveness to $P$. berghei and $P$. yoelii infection by $\sim 77 \%$ and $\sim 34 \%$, respectively (Figure 3C). Sporozoite entry was reduced by $\sim 69 \%$ and $\sim 30 \%$ for $P$. berghei and $P$. yoelii sporozoites, respectively (Figure 3D). Similar to P. falciparum, AQP9 silencing had no effect on cell traversal (Supplementary Figure 9B). P. berghei schizont diameters measured two days post-infection were similar in AQP9-knockout and wild type hepatocytes (Supplementary Figure 10B) thus confirming that AQP9 is not involved in parasite development at the liver stage.
Altogether, these results indicate that $P$. berghei and $P$. yoelii display differential dependence on AQP9 for entry into hepatocytes. Indeed, $P$. yoelii invasion is found to not be or be poorly AQP9-dependent, whereas for P. berghei, invasion depends mainly on AQP9, at least in human liver cells that express this protein.

\section{DISCUSSION}

The first multiplicative stage of malaria parasites within their human host occurs exclusively in the hepatocyte in vivo, and this development can also be obtained in vitro. It is interesting that CD81-expressing human hepatoma HepG2 cells are permissive to productive infection by sporozoites of the rodent parasites $P$. berghei and $P$. yoelii, but not to productive infection by $P$. falciparum (Silvie et al., 2006a). This differential susceptibility was exploited to search for proteins responsible for the exclusive permissiveness of human hepatocytes to $P$. falciparum sporozoites. Comparison of the transcriptome of human hepatocytes with that of human hepatoma HepG2-CD81 cells, which do not support $P$. falciparum sporozoite infection, led to identification of genes whose expression is downregulated in hepatoma cells. We focused our functional studies on AQP9, a membrane protein already suspected to be involved in the virulence of the rodent parasite $P$. berghei (Liu et al., 2007) and whose gene expression was one of those most downregulated in hepatoma cells. Our results demonstrate that AQP9 is required for efficient $P$. falciparum sporozoite entry into human hepatocytes. Furthermore, we found that the rodent parasites $P$. yoelii and $P$. berghei differentially depend on AQP9 and that this difference varies depending on the host cell type.

AQP9 is a member of the AQPs family, which consists of 13 distinct small hydrophobic membrane proteins with predominant roles in trans-cellular water transport in response to osmotic gradients (Verkman, 2005). Among the aquaporins, AQPs 3, 7, 9 and 10, referred to as aquaglyceroporins, also transport glycerol and other small uncharged solutes (Verkman, 2005). Human AQP9 is widely expressed in the liver but also in other tissues, including the lung, spleen and leukocytes, whereas rodent AQP9 has mainly been found at the basolateral hepatocyte plasma membrane facing the sinusoids (Huebert et al., 2002; Mazzone et al., 2006) and in a less abundant manner in other tissues, such as the testis, epididymis, spleen,

TABLE 1 | Summary of the role of AQP9 in susceptibility to Plasmodium infection according to the different cell types and plasmodial species tested on the basis of RNA silencing experiments.

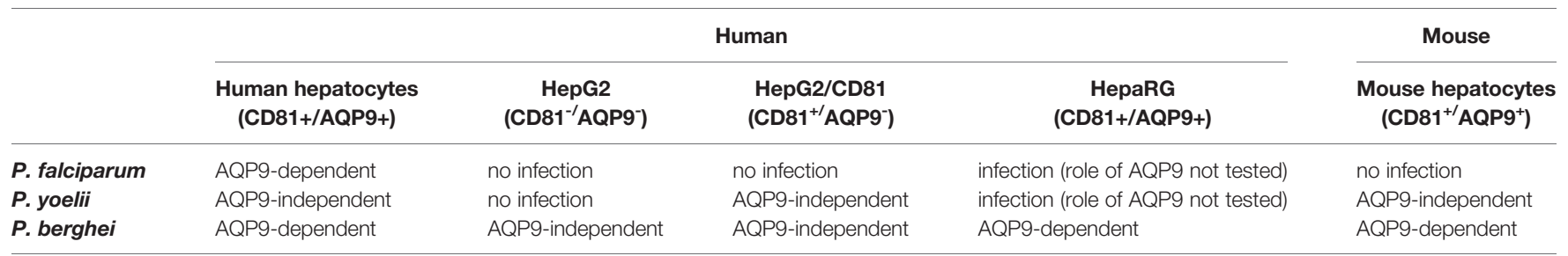


brain and lung (Ishibashi et al., 1998; Tsukaguchi et al., 1998; Elkjaer et al., 2000). By regulating water entry or entry of other small uncharged solutes and interacting with cytoskeleton elements and signalling cascades, the AQPs can influence cell morphology, volume, motility and migration (Saadoun et al., 2005; Holm et al., 2016). Therefore, they are considered potential therapeutic targets (Saadoun et al., 2005).

The results of our gene silencing experiments demonstrate that AQP9 expression is required for P. falciparum sporozoite infection of human hepatocytes, and this requirement appears to be of the same order of magnitude as it is for CD81, which thus far is the only hepatocyte surface molecule known to be strictly required for $P$. falciparum sporozoite infectivity (Silvie et al., 2003). Moreover, overexpression of AQP9, as well as of CD81, in human hepatocytes led to increased $P$. falciparum infection. From our previous studies on CD81 (Silvie et al., 2003) and the current results presented in this paper, one can conclude that P. falciparum require both CD81 and AQP9 to invade hepatocytes as the invasion is drastically hampered in the absence of one or other. There are two arguments that support this conclusion. First, diminishing expression levels of one does not impact on the other one. Second, the reduction in hepatocyte infectivity observed with the inhibition of the expression of either protein was proportional to its inhibition level, as none is able to compensate for silencing of the other, suggesting that these two proteins are operating independently and/or sequentially. While not tested here, it would be interesting to test whether simultaneous silencing of both AQP9 and CD81 proteins could result in a complete invasion-inhibition.

Additional experiments using the rodent parasites $P$. yoelii and $P$. berghei showed that the requirement for AQP9 varies with the species of the parasite and of the host cell. Thus, invasion of $P$. berghei sporozoites was more dependent on the presence of AQP9 than invasion of $P$. yoelii sporozoite in murine hepatocytes, and this effect was more pronounced when human hepatocytes were used. These findings differ from those obtained for CD81 dependence (Silvie et al., 2007). Indeed, when human hepatocytes are used, $P$. yoelii invasion is strictly dependent on CD81 but only poorly dependent on AQP9, whereas for P. berghei, invasion is mainly dependent on AQP9, at least in human liver cells that express this protein, as it is on CD81. It should be noted that $P$. berghei is strictly CD81dependent for invasion of the mouse cell line Hepa 1.6 but CD81-independent when human hepatoma cell lines are used (Silvie et al., 2007).

Taken together, our results showed that AQP9 and CD81 are individually required for Plasmodium sporozoite infection, but that neither is able to compensate for the absence of the other. Moreover, the species specificity for P. falciparum and P. berghei, with a lesser impact on $P$. yoelii, suggest a distinct role for AQP9 to the functions of previously described CD81 or SR-BI (Silvie et al., 2003; Yalaoui et al., 2008a; Risco-Castillo et al., 2014; Manzoni et al., 2017), probably acting downstream to the events requiring both these host proteins. Furthermore, our findings strengthen previous observations that sporozoite entry into liver cells is a complex phenomenon and suggest that extrapolation of results obtained with rodent models to human parasites should be carried out with caution. This complexity is consistent with the general view that entry of intracellular pathogens relies on plastic and dynamic interactions between various molecules at the cell surfaces of the pathogen and host, as illustrated for HIV, HCV, and P. falciparum merozoites (Cosset and Lavillette, 2011; Bartholdson et al., 2013) and for the role of CD81, SR-BI and CD9P-1 in hepatocyte invasion by Plasmodium sporozoites (Yalaoui et al., 2008a; Charrin et al., 2009).

Our experiments with human and rodent parasites showed that AQP9 plays a crucial role in sporozoite invasion but not in the cell traversal that precedes it. Similar results were reported for CD81, which was also found to not be required for migration of P. falciparum and P. yoelii sporozoites through cells (Silvie et al., 2003). Internalization of the Plasmodium sporozoite and the associated PVM formation through invagination of the host cell plasma membrane involves a series of finely orchestrated events, including gliding motility, moving junction formation, and penetration (Dundas et al., 2019). Several studies have shown that this invasion process, as is the case in other Apicomplexa parasites, requires not only the parasite's actin-myosin motor but also membrane reorganization and de novo polymerization of host actin at the entry site for anchoring the junction on which the parasite pulls to penetrate the host cell (Gonzalez et al., 2009; Gomes-Santos et al., 2012; Gaji et al., 2013). Interestingly, previous studies have shown that AQP9 plays a major role in processes involving dynamic membrane reorganization and actin polymerization (Loitto et al., 2002; Loitto et al., 2007). More importantly, studies of AQP9 inhibition have shown that it promotes cell membrane protrusions through its water transport function. It has been proposed that AQP9-induced water influx results in a pressure-driven forward push of the membrane, creating a gap that allows diffusion and polymerization of actin monomers (Loitto et al., 2009; Karlsson et al., 2013). In our study, the inhibitory effect observed with phloretin strongly suggests an important role for the transport of water, and possibly of other small molecules, in liver cell invasion by Plasmodium sporozoites. Although it is not clear how AQP9 functions to promote sporozoite invasion into hepatocytes, one could speculate that AQP9 might play an indirect role through actin polymerization, possibly through its water transport function. Interestingly, a similar mechanism has been reported for Cryptosporidium parvum, another intracellular apicomplexan parasite, in which the host aquaporin AQP1 has been shown to be crucial for entry of the parasite into cholangiocytes (Chen et al., 2005). More specifically, it was shown that AQP1 mediates the localized water influx that leads to the increase in cell volume and actin polymerization accompanying the membrane protrusion that allows C. parvum entry and PVM formation (Chen et al., 2005). Collectively, these data support a model in which AQP9, through its water permeation function and possibly permeation of other small molecules, induces the membrane reorganization and actin polymerization, likely at the entry site, required to permit invagination of the PVM and entry of the sporozoite. Additional studies will be necessary to confirm this hypothesis. 
In conclusion, the present study demonstrates that the host protein AQP9 is involved in entry of $P$. falciparum (and of $P$. berghei) sporozoites into hepatocytes and suggests that this process may involve water transport and possibly other small molecules. Our findings also suggest that P. falciparum sporozoites are able to highjack hepatocyte membrane machinery to achieve internalization into the parasitophorous vacuole. Collectively, our data provide new insights into the complex cellular process required for Plasmodium infection of liver cells and may assist in the development of host factordirected antimalarial therapies.

\section{DATA AVAILABILITY STATEMENT}

The original contributions presented in the study are included in the article/Supplementary Material. Further inquiries can be directed to the corresponding author.

\section{ETHICS STATEMENT}

The protocol was approved by the Ethics Committee for Animal Experiments of University Pierre et Marie Curie, Paris 6, France (Permit Number: 75-1087).

\section{AUTHOR CONTRIBUTIONS}

NA, J-FF, ST, SY, PF, and DM conceived, planned, and designed experiments. NA, J-FF, ST, SY, AL, VS, AR, AG, VR-C and MT conducted experiments. NA, J-FF, ST, SY, AL, VS, AG, AR, VR-C, AM, MT, G-JG, RS, J-CV, PR, J-LP, PF, and DM analyzed the data. J-FF, PF, G-JG, RS, J-CV, PR and DM provided essential materials. NA, J-FF, ST, SY, PF, J-LP and DM wrote the manuscript. All authors contributed to the article and approved the submitted version.

\section{REFERENCES}

Bartholdson, S. J., Crosnier, C., Bustamante, L. Y., Rayner, J. C., and Wright, G. J. (2013). Identifying Novel Plasmodium Falciparum Erythrocyte Invasion Receptors Using Systematic Extracellular Protein Interaction Screens. Cell. Microbiol. 15, 1304-1312. doi: 10.1111/cmi.12151

Bartosch, B., Vitelli, A., Granier, C., Goujon, C., Dubuisson, J., Pascale, S., et al. (2003). Cell Entry of Hepatitis C Virus Requires a Set of Co-Receptors That Include the CD81 Tetraspanin and the SR-B1 Scavenger Receptor. J. Biol. Chem. 278, 41624-41630. doi: 10.1074/jbc.M305289200

Castaing, M., Guerci, A., Mallet, J., Czernichow, P., Ravassard, P., and Scharfmann, R. (2005). Efficient Restricted Gene Expression in Beta Cells by Lentivirus-Mediated Gene Transfer Into Pancreatic Stem/Progenitor Cells. Diabetologia 48, 709-719. doi: 10.1007/s00125-005-1694-6

Ceelen, L., De Spiegelaere, W., David, M., De Craene, J., Vinken, M., Vanhaecke, T., et al. (2011). Critical Selection of Reliable Reference Genes for Gene Expression Study in the HepaRG Cell Line. Biochem. Pharmacol. 81, 12551261. doi: 10.1016/j.bcp.2011.03.004

Charrin, S., Yalaoui, S., Bartosch, B., Cocquerel, L., Franetich, J.-F., Boucheix, C., et al. (2009). The Ig Domain Protein CD9P-1 Down-Regulates CD81 Ability to

\section{FUNDING}

SY was supported by a fellowship from the European Community. This work was supported in part by grants from the Agence Nationale pour la Recherche (ANR-6 BLAN-0378 and MaTuRe project) and the European Community (LSHP-CT2005-012199/MALINV).

\section{ACKNOWLEDGMENTS}

The authors are indebted to Dr. Olivier Silvie for fruitful discussions and critical reading of the manuscript and to Dr. Georges Snounou for fruitful discussions. We are grateful to $\mathrm{Mr}$. Thierry Houpert for his expertise and precious technical assistance with the maintenance of the Anopheles stephensi colony. Microarray hybridization and analysis were performed at the "Plateforme post-génomique de la Pitié-Salpêtrière". We would like to thank Perrine Frere and Aurélien Dauphin for confocal microscopy assistance in the imaging facilities "Plateforme d'Imagerie Cellulaire Pitié-Salpêtrière (Shared Resource Facilities of CRICM, IFR14, IFR113, INSERM, UPMC). We also thank Catherine Blanc and Bénédicte Hoareau for flow cytometry assistance (Flow Cytometry Core CyPS, Université Pierre et Marie Curie \& Groupe hospitalier Pitié-Salpêtrière).

In memory of Patrick Froissard who tragically left us during the course of this study.

\section{SUPPLEMENTARY MATERIAL}

The Supplementary Material for this article can be found online at: https://www.frontiersin.org/articles/10.3389/fcimb.2021.704662/ full\#supplementary-material

Support Plasmodium Yoelii Infection. J. Biol. Chem. 284, 31572-31578. doi: 10.1074/jbc.M109.057927

Chen, X.-M., O'Hara, S. P., Huang, B. Q., Splinter, P. L., Nelson, J. B., and LaRusso, N. F. (2005). Localized Glucose and Water Influx Facilitates Cryptosporidium Parvum Cellular Invasion by Means of Modulation of Host-Cell Membrane Protrusion. Proc. Natl. Acad. Sci. U. S. A. 102, 6338-6343. doi: 10.1073/ pnas.0408563102

Cosset, F.-L., and Lavillette, D. (2011). Cell Entry of Enveloped Viruses. Adv. Genet. 73, 121-183. doi: 10.1016/B978-0-12-380860-8.00004-5

Dundas, K., Shears, M. J., Sinnis, P., and Wright, G. J. (2019). Important Extracellular Interactions Between Plasmodium Sporozoites and Host Cells Required for Infection. Trends Parasitol. 35, 129-139. doi: 10.1016/ j.pt.2018.11.008

Elkjaer, M., Vajda, Z., Nejsum, L. N., Kwon, T., Jensen, U. B., Amiry-Moghaddam, M., et al. (2000). Immunolocalization of AQP9 in Liver, Epididymis, Testis, Spleen, and Brain. Biochem. Biophys. Res. Commun. 276, 1118-1128. doi: 10.1006/bbrc.2000.3505

Franke-Fayard, B., Trueman, H., Ramesar, J., Mendoza, J., van der Keur, M., van der Linden, R., et al. (2004). A Plasmodium Berghei Reference Line That 
Constitutively Expresses GFP at a High Level Throughout the Complete Life Cycle. Mol. Biochem. Parasitol. 137, 23-33. doi: 10.1016/j.molbiopara.2004. 04.007

Gaji, R. Y., Huynh, M.-H., and Carruthers, V. B. (2013). A Novel High Throughput Invasion Screen Identifies Host Actin Regulators Required for Efficient Cell Entry by Toxoplasma Gondii. PloS One 8, e64693. doi: 10.1371/ journal.pone. 0064693

Gego, A., Silvie, O., Franetich, J.-F., Farhati, K., Hannoun, L., Luty, A. J. F., et al. (2006). New Approach for High-Throughput Screening of Drug Activity on Plasmodium Liver Stages. Antimicrob. Agents Chemother. 50, 1586-1589. doi: 10.1128/AAC.50.4.1586-1589.2006

Gomes-Santos, C. S. S., Itoe, M. A., Afonso, C., Henriques, R., Gardner, R., Sepúlveda, N., et al. (2012). Highly Dynamic Host Actin Reorganization Around Developing Plasmodium Inside Hepatocytes. PloS One 7, e29408. doi: 10.1371/journal.pone.0029408

Gonzalez, V., Combe, A., David, V., Malmquist, N. A., Delorme, V., Leroy, C., et al. (2009). Host Cell Entry by Apicomplexa Parasites Requires Actin Polymerization in the Host Cell. Cell Host Microbe 5, 259-272. doi: 10.1016/ j.chom.2009.01.011

Holm, A., Magnusson, K.-E., and Vikström, E. (2016). Pseudomonas Aeruginosa N-3-Oxo-Dodecanoyl-Homoserine Lactone Elicits Changes in Cell Volume, Morphology, and AQP9 Characteristics in Macrophages. Front. Cell. Infect. Microbiol. 6, 32. doi: 10.3389/fcimb.2016.00032

Huebert, R. C., Splinter, P. L., Garcia, F., Marinelli, R. A., and LaRusso, N. F. (2002). Expression and Localization of Aquaporin Water Channels in Rat Hepatocytes. Evidence for a Role in Canalicular Bile Secretion. J. Biol. Chem. 277, 22710-22717. doi: 10.1074/jbc.M202394200

Ishibashi, K., Kuwahara, M., Gu, Y., Tanaka, Y., Marumo, F., and Sasaki, S. (1998). Cloning and Functional Expression of a New Aquaporin (AQP9) Abundantly Expressed in the Peripheral Leukocytes Permeable to Water and Urea, But Not to Glycerol. Biochem. Biophys. Res. Commun. 244, 268-274. doi: 10.1006/bbrc.1998.8252

Isom, H. C., Secott, T., Georgoff, I., Woodworth, C., and Mummaw, J. (1985). Maintenance of Differentiated Rat Hepatocytes in Primary Culture. Proc. Natl. Acad. Sci. U. S. A. 82, 3252-3256. doi: 10.1073/pnas.82.10.3252

Karlsson, T., Bolshakova, A., Magalhães, M. A. O., Loitto, V. M., and Magnusson, K.-E. (2013). Fluxes of Water Through Aquaporin 9 Weaken MembraneCytoskeleton Anchorage and Promote Formation of Membrane Protrusions. PloS One 8, e59901. doi: 10.1371/journal.pone.0059901

Liu, Y., Promeneur, D., Rojek, A., Kumar, N., Frøkiaer, J., Nielsen, S., et al. (2007). Aquaporin 9 is the Major Pathway for Glycerol Uptake by Mouse Erythrocytes, With Implications for Malarial Virulence. Proc. Natl. Acad. Sci. U. S. A. 104, 12560-12564. doi: 10.1073/pnas.0705313104

Loitto, V.-M., Forslund, T., Sundqvist, T., Magnusson, K.-E., and Gustafsson, M. (2002). Neutrophil Leukocyte Motility Requires Directed Water Influx. J. Leukoc. Biol. 71, 212-222.

Loitto, V. M., Huang, C., Sigal, Y. J., and Jacobson, K. (2007). Filopodia Are Induced by Aquaporin-9 Expression. Exp. Cell Res. 313, 1295-1306. doi: 10.1016/j.yexcr.2007.01.023

Loitto, V. M., Karlsson, T., and Magnusson, K.-E. (2009). Water Flux in Cell Motility: Expanding the Mechanisms of Membrane Protrusion. Cell Motil. Cytoskeleton 66, 237-247. doi: 10.1002/cm.20357

Manzoni, G., Marinach, C., Topçu, S., Briquet, S., Grand, M., Tolle, M., et al. (2017). Plasmodium P36 Determines Host Cell Receptor Usage During Sporozoite Invasion. eLife 6, e25903. doi: 10.7554/eLife.25903

Mazzone, A., Tietz, P., Jefferson, J., Pagano, R., and LaRusso, N. F. (2006). Isolation and Characterization of Lipid Microdomains From Apical and Basolateral Plasma Membranes of Rat Hepatocytes. Hepatol. Baltim. Md. 43, 287-296. doi: 10.1002/hep.21039

Mosmann, T. (1983). Rapid Colorimetric Assay for Cellular Growth and Survival: Application to Proliferation and Cytotoxicity Assays. J. Immunol. Methods 65, 55-63. doi: 10.1016/0022-1759(83)90303-4

Mota, M. M., Pradel, G., Vanderberg, J. P., Hafalla, J. C., Frevert, U., Nussenzweig, R. S., et al. (2001). Migration of Plasmodium Sporozoites Through Cells Before Infection. Science 291, 141-144. doi: 10.1126/science.291.5501.141

Posfai, D., Maher, S. P., Roesch, C., Vantaux, A., Sylvester, K., Péneau, J., et al. (2020). Plasmodium Vivax Liver and Blood Stages Recruit the Druggable Host Membrane Channel Aquaporin-3. Cell Chem. Biol. 27, 719-727. doi: 10.1016/ j.chembiol.2020.03.009
Posfai, D., Sylvester, K., Reddy, A., Ganley, J. G., Wirth, J., Cullen, Q. E., et al. (2018). Plasmodium Parasite Exploits Host Aquaporin-3 During Liver Stage Malaria Infection. PloS Pathog. 14, e1007057. doi: 10.1371/journal.ppat.1007057

Prudêncio, M., Rodrigues, C. D., Ataíde, R., and Mota, M. M. (2008). Dissecting In Vitro Host Cell Infection by Plasmodium Sporozoites Using Flow Cytometry. Cell. Microbiol. 10, 218-224. doi: 10.1111/j.1462-5822.2007.01032.x

Rénia, L., Miltgen, F., Charoenvit, Y., Ponnudurai, T., Verhave, J. P., Collins, W. E., et al. (1988). Malaria Sporozoite Penetration. A New Approach by Double Staining. J. Immunol. Methods 112, 201-205. doi: 10.1016/0022-1759(88)90358-4

Risco-Castillo, V., Topçu, S., Son, O., Briquet, S., Manzoni, G., and Silvie, O. (2014). CD81 Is Required for Rhoptry Discharge During Host Cell Invasion by Plasmodium Yoelii Sporozoites. Cell. Microbiol. 16, 1533-1548. doi: 10.1111/ cmi. 12309

Rodrigues, C. D., Hannus, M., Prudêncio, M., Martin, C., Gonçalves, L. A., Portugal, S., et al. (2008). Host Scavenger Receptor SR-BI Plays a Dual Role in the Establishment of Malaria Parasite Liver Infection. Cell Host Microbe 4, 271-282. doi: 10.1016/j.chom.2008.07.012

Rojek, A. M., Skowronski, M. T., Füchtbauer, E.-M., Füchtbauer, A. C., Fenton, R. A., Agre, P., et al. (2007). Defective Glycerol Metabolism in Aquaporin 9 (AQP9) Knockout Mice. Proc. Natl. Acad. Sci. U. S. A. 104, 3609-3614. doi: 10.1073/ pnas.0610894104

Russ, H. A., Bar, Y., Ravassard, P., and Efrat, S. (2008). In Vitro Proliferation of Cells Derived From Adult Human Beta-Cells Revealed by Cell-Lineage Tracing. Diabetes 57, 1575-1583. doi: 10.2337/db07-1283

Saadoun, S., Papadopoulos, M. C., Hara-Chikuma, M., and Verkman, A. S. (2005). Impairment of Angiogenesis and Cell Migration by Targeted Aquaporin-1 Gene Disruption. Nature 434, 786-792. doi: 10.1038/nature03460

Silvie, O., Charrin, S., Billard, M., Franetich, J.-F., Clark, K. L., van Gemert, G.-J., et al. (2006a). Cholesterol Contributes to the Organization of TetraspaninEnriched Microdomains and to CD81-Dependent Infection by Malaria Sporozoites. J. Cell Sci. 119, 1992-2002. doi: 10.1242/jcs.02911

Silvie, O., Franetich, J.-F., Boucheix, C., Rubinstein, E., and Mazier, D. (2007). Alternative Invasion Pathways for Plasmodium Berghei Sporozoites. Int. J. Parasitol. 37, 173-182. doi: 10.1016/j.ijpara.2006.10.005

Silvie, O., Greco, C., Franetich, J.-F., Dubart-Kupperschmitt, A., Hannoun, L., van Gemert, G.-J., et al. (2006b). Expression of Human CD81 Differently Affects Host Cell Susceptibility to Malaria Sporozoites Depending on the Plasmodium Species. Cell. Microbiol. 8, 1134-1146. doi: 10.1111/j.1462-5822.2006.00697.x

Silvie, O., Rubinstein, E., Franetich, J.-F., Prenant, M., Belnoue, E., Rénia, L., et al. (2003). Hepatocyte CD81 is Required for Plasmodium Falciparum and Plasmodium Yoelii Sporozoite Infectivity. Nat. Med. 9, 93-96. doi: 10.1038/ nm808

Silvie, O., Semblat, J. P., Franetich, J. F., Hannoun, L., Eling, W., and Mazier, D. (2002). Effects of Irradiation on Plasmodium Falciparum Sporozoite Hepatic Development: Implications for the Design of Pre-Erythrocytic Malaria Vaccines. Parasite Immunol. 24, 221-223. doi: 10.1046/j.1365-3024.2002.00450.x

Tsukaguchi, H., Shayakul, C., Berger, U. V., Mackenzie, B., Devidas, S., Guggino, W. B., et al. (1998). Molecular Characterization of a Broad Selectivity Neutral Solute Channel. J. Biol. Chem. 273, 24737-24743. doi: 10.1074/jbc.273. 38.24737

van Schaijk, B. C. L., Janse, C. J., van Gemert, G.-J., van Dijk, M. R., Gego, A., Franetich, J.-F., et al. (2008). Gene Disruption of Plasmodium Falciparum p52 Results in Attenuation of Malaria Liver Stage Development in Cultured Primary Human Hepatocytes. PloS One 3, e3549. doi: 10.1371/journal.pone.0003549

Verkman, A. S. (2005). More Than Just Water Channels: Unexpected Cellular Roles of Aquaporins. J. Cell Sci. 118, 3225-3232. doi: 10.1242/jcs.02519

WHO | World Malaria Report 2018 WHO. Available at: http://www.who.int/ malaria/publications/world-malaria-report-2018/en/ (Accessed June 4, 2020).

Yalaoui, S., Huby, T., Franetich, J.-F., Gego, A., Rametti, A., Moreau, M., et al. (2008a). Scavenger Receptor BI Boosts Hepatocyte Permissiveness to Plasmodium Infection. Cell Host Microbe 4, 283-292. doi: 10.1016/ j.chom.2008.07.013

Yalaoui, S., Zougbédé, S., Charrin, S., Silvie, O., Arduise, C., Farhati, K., et al. (2008b). Hepatocyte Permissiveness to Plasmodium Infection Is Conveyed by a Short and Structurally Conserved Region of the CD81 Large Extracellular Domain. PloS Pathog. 4, e1000010. doi: 10.1371/journal.ppat.1000010

Yang, B., and Verkman, A. S. (2002). Analysis of Double Knockout Mice Lacking Aquaporin-1 and Urea Transporter UT-B. Evidence for UT-B-Facilitated 
Water Transport in Erythrocytes. J. Biol. Chem. 277, 36782-36786. doi: 10.1074/jbc.M206948200

Yee, J. K., Miyanohara, A., LaPorte, P., Bouic, K., Burns, J. C., and Friedmann, T. (1994). A General Method for the Generation of High-Titer, Pantropic Retroviral Vectors: Highly Efficient Infection of Primary Hepatocytes. Proc. Natl. Acad. Sci. U. S. A. 91, 9564-9568. doi: 10.1073/pnas.91.20.9564

Zennou, V., Petit, C., Guetard, D., Nerhbass, U., Montagnier, L., and Charneau, P. (2000). HIV-1 Genome Nuclear Import is Mediated by a Central DNA Flap. Cell 101, 173-185. doi: 10.1016/S0092-8674(00)80828-4

Zufferey, R., Nagy, D., Mandel, R. J., Naldini, L., and Trono, D. (1997). Multiply Attenuated Lentiviral Vector Achieves Efficient Gene Delivery In Vivo. Nat. Biotechnol. 15, 871-875. doi: 10.1038/nbt0997-871
Conflict of Interest: The authors declare that the research was conducted in the absence of any commercial or financial relationships that could be construed as a potential conflict of interest.

Copyright (c) 2021 Amanzougaghene, Tajeri, Yalaoui, Lorthiois, Soulard, Gego, Rametti, Risco-Castillo, Moreno, Tefit, van Gemert, Sauerwein, Vaillant, Ravassard, Pérignon, Froissard, Mazier and Franetich. This is an open-access article distributed under the terms of the Creative Commons Attribution License (CC BY). The use, distribution or reproduction in other forums is permitted, provided the original author(s) and the copyright owner(s) are credited and that the original publication in this journal is cited, in accordance with accepted academic practice. No use, distribution or reproduction is permitted which does not comply with these terms. 